\title{
Studies on Water Control by Filtration through Sand Bed in a Marine Aquarium with Closed Circulating System-VI. Acidification of Aquarium Water
}

\author{
Kazutsugu Hirayama* \\ (Received October 14, 1969)
}

In previous papers ${ }^{1,2,3,4)}$, it was experimentally confirmed that in marine aquariums with closed circulating system, the oxygen consumption during filtration could be used as an excellent index of the degree of purification of culture water by filtration, and this index was used to investigate the balance between purification and pollution of the culture water as well as to determine the capacity of marine aquarium against the number and total weight of fishes to be cultured therein.

Although the culture water polluted by excretion of fishes and by feeding may be purified by filtration through sand bed, it tends to show some characteristic changes in chemical composition along with the lapse of time. According to SAEKI ${ }^{5)}$, the major changes are remarkable accumulation of nitrate as the result of nitrification on the filter sands, and reduced alkalinity and increased hydrogen ion concentration due to the decrease of alkali (magnesium) and the increase of acid (nitrate).

KaWAI et al..$^{6)}$ pointed out that the nitrifying activity of filter sands in marine aquariums with closed circulating system was maximum at the $\mathrm{pH}$ value of 9.0 and it decreased with the increase of hydrogen ion concentration.

In spite of many studies on aquarium with closed circulating system, the relations of acidifying velocity of the aquarium water to the weight of feed per day and to the body weight of fish cultured in the aquarium have heretofore been left unexplored. In addition, the changes in chemical composition of the aquarium sea water following acidification have been left as the important problems to be solved. Moreover, to keep fish healthy, the physiological effects of those changes on fish must also be clarified. In order to solve these problems, the present study was performed experimentally by culturing fish in experimental aquariums.

\section{Methods and Materials}

The experiments were carried out under the culture conditions as shown in Table 1, by using experimental aquariums made of vinyl chloride plate as shown in Fig. 1. Three types of filter sands varying in grain size were used in the experiments. The grain size

\footnotetext{
* Faculty of Fisheries, Nagasaki University, Nagasaki, Japan. (平山和次：長崎大学水産学部)
} 
Table 1. Culture conditions.

Water

\begin{tabular}{c|c|c|c}
\hline $\begin{array}{c}\text { Specific gravity } \\
\left(15^{\circ} \mathrm{C}\right)\end{array}$ & Water temp. & $\begin{array}{c}\text { Circulating } \\
\text { velocity }\end{array}$ & Volume \\
\hline About 1.023 & $23^{\circ} \mathrm{C}-25^{\circ} \mathrm{C}$ & $2 l / \mathrm{min}$. & $50 l$ \\
\hline
\end{tabular}

Filter

\begin{tabular}{c|c|c}
\hline Surface dia. & Bottom dia. & Depth \\
\hline $21 \mathrm{~cm}$ & $19 \mathrm{~cm}$ & $16.5 \mathrm{~cm}$ \\
\hline
\end{tabular}

Filter sands

\begin{tabular}{|c|c|c|c|}
\hline $\begin{array}{c}\text { Filter } \\
\text { number }\end{array}$ & $\begin{array}{l}\text { Representative } \\
\text { diameter }\end{array}$ & $\begin{array}{c}\text { Mesh of } \\
\text { sieve }\end{array}$ & $\begin{array}{l}\text { Filtration } \\
\text { Rate }(\%)\end{array}$ \\
\hline No. 1 & $\begin{array}{l}2.1 \mathrm{~mm} \\
\text { (Grain size coeff. } \\
\quad: \quad 47.2 \text { ) }\end{array}$ & $\begin{array}{l}2 \mathrm{~mm} \\
3 \mathrm{~mm} \\
4 \mathrm{~mm}\end{array}$ & $\begin{array}{r}39.27 \\
99.86 \\
100\end{array}$ \\
\hline No. 2 & $\begin{array}{l}3.9 \mathrm{~mm} \\
\text { (Grain size coeff. } \\
: \quad 25.7)\end{array}$ & $\begin{array}{l}3 \mathrm{~mm} \\
4 \mathrm{~mm} \\
5 \mathrm{~mm}\end{array}$ & $\begin{array}{r}0.96 \\
58.36 \\
100\end{array}$ \\
\hline No. 3 & $\begin{array}{l}8.3 \mathrm{~mm} \\
\text { (Grain size coeff. } \\
\quad: \quad 12.0 \text { ) }\end{array}$ & $\begin{array}{r}7 \mathrm{~mm} \\
8 \mathrm{~mm} \\
9 \mathrm{~mm} \\
10 \mathrm{~mm}\end{array}$ & $\begin{array}{r}5.96 \\
38.20 \\
72.37 \\
100\end{array}$ \\
\hline
\end{tabular}

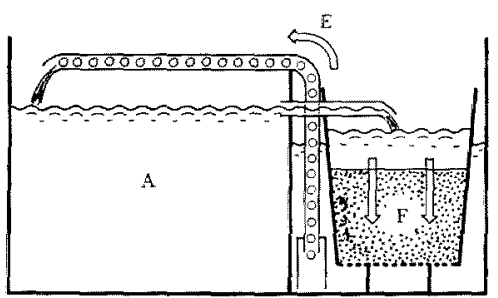

Fig. 1. Experimental aquarium.

A: Culture tank. F: Filter. E: Exhausting device. $\diamond$ : Flow of water.

coefficient shown in Table 1 indicates the reciprocal number of the representative diameter of grains of filter sands. Further details of this index may be referred to the present author's previous paper $^{3}$. Filter sands were washed thoroughly with concentrated hydrochloric acid before experiments in order to eliminate $\mathrm{CaCO}_{3}$ such as coral and shell.

The fish used in the experiments was black sea bream, Mylio macrophalus, fed with shrimp, except in the experiments on the effect of adding $\mathrm{CaCO}_{3}$ to the aquarium on the prevention of acidification of sea water in which "ISHIDAI" (Japanese name), Oplegnathus fasciatus, was cultured by feeding with fish.

Sample water was filtered with membrane filter (pore: $400 \mathrm{~m} \mu$ ) before analysis. Analytical methods of sea water are as follows.

$\mathrm{pH}$ : Glass electrode method. Alkalinity: Titration method by $1 / 100 \mathrm{~N} \mathrm{HCl}$ solution with BCP. Ammonium-N: Diffusion method slightly modified by the author. NitriteN: Colorimetric method by G. R. reagent. Nitrate-N: Colorimetric method by reduction to nitrite by hydrazine sulfate. 
Phosphate-P: Denigès-Atkins method. Calcium: Titration method by EDTA solution. COD: Oxidation method with alkali $(\mathrm{NaOH})$.

\section{Results}

Relations of acidifying velocity of aquarium water to feed weight a day and to body weight of fish Four black sea breams kept in the experimental aquarium provided with filter of enough purifying ability were fed daily with a definite weight of shrimp. The body weight of black sea breams used in the experiments ranged from $20 \mathrm{~g}$ to $48 \mathrm{~g}$, and the total weight of four fishes used in various experiments was 99.8-149.6 g.

The courses of acidification of the aquarium water obtained from daily measurements of alkalinity are represented in Fig. 2. Each course of decrease of alkalinity is quite linear until it reaches 0.2 meq. $/ l$, except for the cases of no feeding.

The experiments in which four fishes were fed at the rate of $5 \mathrm{~g}$ and $10 \mathrm{~g}$ per day were performed with three aquariums having filters varying in grain size of sands. The results of those experiments are also shown in Fig. 2. The courses of decrease of alkalinity were all the same in three aquariums in spite of the difference in grain size of filter sands. Accordingly, it was confirmed that acidifying velocity of the aquarium sea water was not affected by the grain size of filter sands.

The equivalent value of acidification per day was calculated from the volume of the

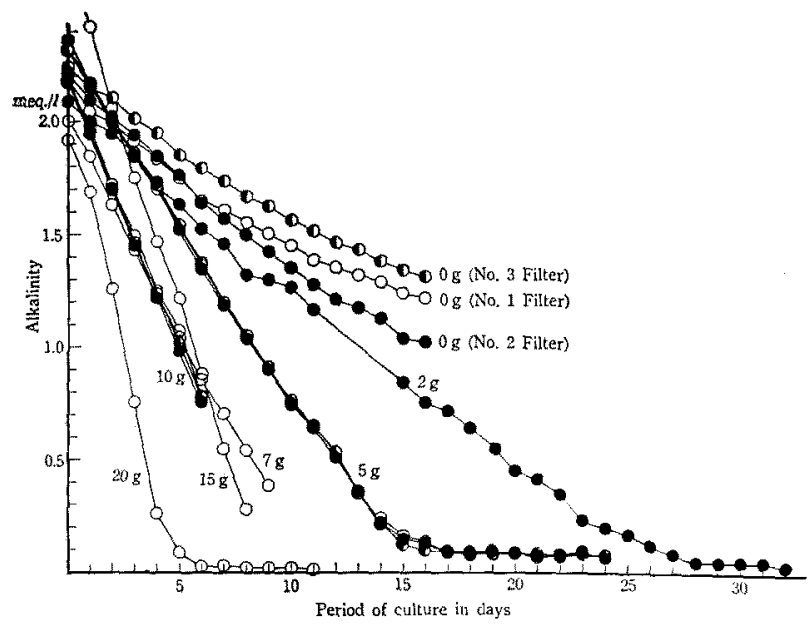

Fig. 2. Courses of decrease in alkalinity of the aquarium water in which fishes were cultured by feeding with shrimp varying in weight per day by various experiments.

$O$ : Aquarium with No. 1 filter sands.

- : Aquarium with No. 2 filter sands.

D: Aquarium with No. 3 filter sands.

The figure with $g$ shown along each line represents the weight (gram) of feed per day. 


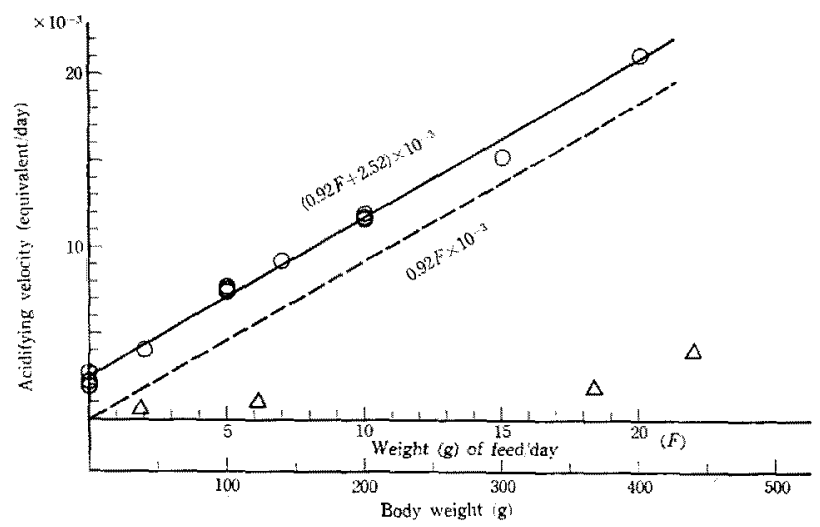

Fig. 3. Relations of the acidifying velocity of the aquarium water to the feeding rate and to the body weight of fish. $O$ : Relation to the feeding rate. $\triangle$ : Relation to the body weight.

aquarium water and from the gradient of linear regression fitted to the daily values of alkalinity. The relation between the degree of acidification thus obtained and the feeding rate is shown in Fig. 3. The relation is quite linear and its equation is shown as

$$
V=(0.92 F+2.52) 10^{-3},
$$

where $V$ and $F$ are the equivalent value of acidification per day (acidifying velocity) and the weight $(\mathrm{g})$ of feed per day, respectively. In the equation, $2.52 \times 10^{-3}$ represents the acidifying velocity attributable to the standard metabolism of four fishes, and $0.92 \times$ $10^{-3}$ represents the increase of the equivalent value of acidification per day, accompanied with the increase of $1 \mathrm{~g}$ in the rate of feed weight per day.

The relation between the acidifying velocity of the aquarium water (eq./day) attributable to standard metabolism and the body weight of cultured fish was obtained by measuring alkalinity once a day during the course of culturing black sea breams of various body weights in the experimental aquarium without feeding. As shown in Fig. 2, the results of experiments in which four fishes (body weight: $23.8-45.2 \mathrm{~g}$, total: $141.7-$ $149.6 \mathrm{~g}$ ) were kept in each of three aquariums with different filter sands indicated that the acidifying velocity of the aquarium water decreases along with the elongation of culture period. However, from the 10 th day after the commencement of culture, the acidifying velocity became almost constant regardless of the period of culture. Hence, the gradient of linear regression representing the course of decrease of alkalinity was calculated from the daily values after the 10th day when the acidifying velocity became almost constant. The equivalent value of acidification per day was estimated from the volume of aquarium water and from the gradient thus obtained, and it is shown in Fig. 3. Although the number of experiments was not sufficient to explain definitely the relation between the acidifying velocity of the aquarium water and the body weight of the fish cultured in the aquarium, 
the results obtained suggested that the variation of the body weight, as compared with that of feeding rate, might not cause any significant difference to the acidifying velocity of aquarium sea water. Although the individual body weight and the total weight of four fishes used in the experiments for the effect of feeding rate on the acidifying velocity varied to some extent, the effect of this extent of variation in body weight of fishes on the acidifying velocity was negligible in these experiments as known from the above-mentioned fact.

Changes in chemical composition of aquarium water accompanied with Reduction of pH value and alkalinity and harmful influences on cultured fish In order to investigate the changes in chemical composition of aquarium sea water accompanied with the reduction of $\mathrm{pH}$ value and alkalinity value, some chemical components in the aquarium water in which four fishes (body weight: $32.6 \mathrm{~g}-36.1 \mathrm{~g}$, total: $139.7 \mathrm{~g}$ ) were cultured by feeding at the rate of $2 \mathrm{~g}$ per day were determined during the entire period of culture. The experiment was carried out with the aquarium having the filter of full purifying ability, after replacing the aquarium water by new sea water. The results obtained are shown in Fig. 4. During 24 days after beginning the experiment, the value of alkalinity decreased linearly from 2.1 meq. $/ l$ to about 0.2 meq. $/ l$. As the alkalinity value reached near 0.2 meq. $/ l$, the rate of its decrease began to reduce and ammonium- $\mathrm{N}$ which had remained under $0.2 \mathrm{ppm}$ began rapid accumulation. The fact that accumulation of ammonium$\mathrm{N}$ and decrease of acidifying velocity could not be observed at the $\mathrm{pH}$ value under 7.0, suggests that nitrifying ability in the filter is enough to keep ammonium- $\mathrm{N}$ at a low level, even if $\mathrm{pH}$ and alkalinity show

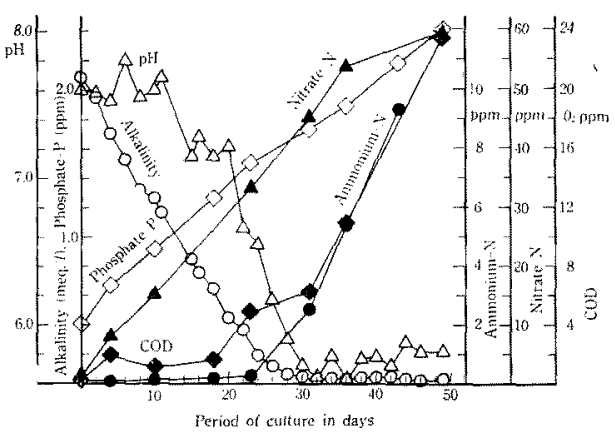

Fig. 4. Changes in chemical composition of aquarium water in which the fishes were cultured with $2 \mathrm{~g}$ of feed per day.

$\bigcirc:$ Alkalinity. $\triangle:$ pH. $\diamond:$ Phosphate-P. $\triangle$ : Nitrate-N. $\bullet:$ Ammonium-N. COD. remarkably low values. Nitrate- $\mathrm{N}$ was accumulated linearly from the beginning of experiment and even after the ceasing of acidification at a low $\mathrm{pH}$ value, the accumulation was continued though at a rate less than that in the water at a high $\mathrm{pH}$ values. Therefore, the nitrifying activity of a filter is restricted only to some extent without ceasing completely even if the $\mathrm{pH}$ value decreases under 6.0 and the value of alkalinity is reduced under 0.05 meq. $/ l$.

The value of COD like in case of ammonium-N started to increase when alkalinity value reached near 0 meq. $/ l$. On the other hand, phosphate-P seemed to be accumulated almost linearly regardless of the reduction of $\mathrm{pH}$ value and alkalinity value. 
In order to ascertain in more detail the facts mentioned above, four fishes (body weight: $28.6 \mathrm{~g}-48.4 \mathrm{~g}$, total: $145.9 \mathrm{~g}$ ) were cultured in experimental aquarium with $20 \mathrm{~g}$ of feed per day, and the changes of chemical components were sought during the entire period of culture. The value of alkalinity was reduced under 0.05 meq. $/ l$ on the 6 th day after the commencement of experiment, but the feeding was still continued until the 25 th day, when fishes were removed and feeding was discontinued while the aquarium water alone continued to circulate. From the 36 th day, sodium bicarbonate was added to the aquarium water in order to keep the $\mathrm{pH}$ value above 7.0 and the alkalnity value above 1.0 meq. $/ l$.

As shown in Fig. 5, the changes in chemical composition of aquarium sea water followed the similar process to those in the experiment with $2 \mathrm{~g}$ of feed per day. Nitrate$\mathrm{N}$ continued to be accumulated in the aquarium water even after the removal of fishes, but ammonium- $\mathrm{N}$ began to decrease after the removal. These facts confirm that nitrification never ceases completely even at the $\mathrm{pH}$ value of 4.8 and the alkalinity value of nearly 0 meq. $l$. Moreover, when sodium bicarbonate was added to the aquarium water, nitrifying activity was rapidly restored.

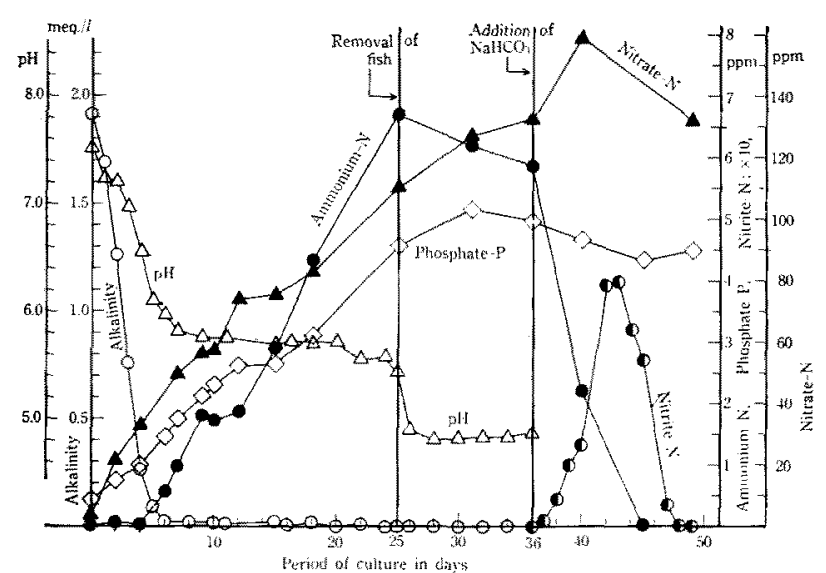

Fig. 5. Changes in chemical composition of aquarium water in which the fishes were cultured with $20 \mathrm{~g}$ of feed per day.

$\bigcirc:$ Alkalinity. $\triangle:$ pH. $\diamond:$ Phosphate-P. $\wedge$ : Nitrate-N, O: Nitrite-N. • : Ammonium-N.

During the course of this experiment, the cultured fish never showed any abnormal symptom, though ammonium- $\mathrm{N}$ accumulated in the aquarium water reached the maximum amount of about $67 \mathrm{ppm}$ with the $\mathrm{pH}$ value of 5.7 and with the alkalinity value of 0.03 meq. $/ l$. It is clarified by this finding that the low values of $\mathrm{pH}$ and alkalinity alone hardly bring forth any harmful influence on the fish and, so far as the $\mathrm{pH}$ value and the alkalinity 
value remain within low levels, ammonium- $\mathrm{N}$ accumulated in the sea water brings little influence.

Prevention of $\mathrm{pH}$ value and alkalinity value from reduction In order to prevent the culture water from quick acidification, $\mathrm{CaCO}_{3}$ such as coral and shell is usually added to the aquarium. Although this method has long been well known, the preventive effect of the dissolution of calcium from coral and shell on acidification has remained unclarified.

The coral $(720 \mathrm{~g}, 370 \mathrm{ml})$ crushed to $5-10 \mathrm{~mm}$ dia. gravels was put on the filter of experimental aquarium, in which two of Oplegnathus weighing about $77 \mathrm{~g}$ each were cultured by feeding with $4 \mathrm{~g}$ of fish per day. The course of change in $\mathrm{pH}$ value, alkalinity value and amount of dissolved calcium obtained by daily measurements was compared with that in the experiment in which two fishes were cultured in the same manner except for the addition of coral (control test.)

The results are shown in Fig. 6. The pH values and the alkalinity values of the sea water in two aquarium decreased through the same process until the 4th day irrespective of the presence of coral. However, from the 4th day, the acidifying velocity of the water

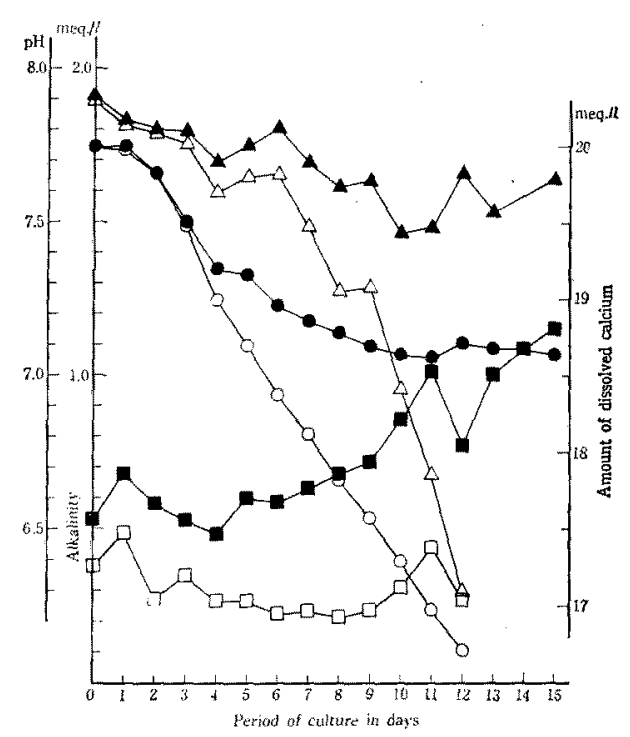

Fig. 6. Preventive effect of coral on acidification of the aquarium water in which the fishes were cultured with $4 \mathrm{~g}$ of feed per day. Closed marks and open marks represent the experiments in the aquariums with and without coral, respectively. Circle, triangle and square represent alkalinity value, $\mathrm{pH}$ value and dissolved calcium content, respectively.

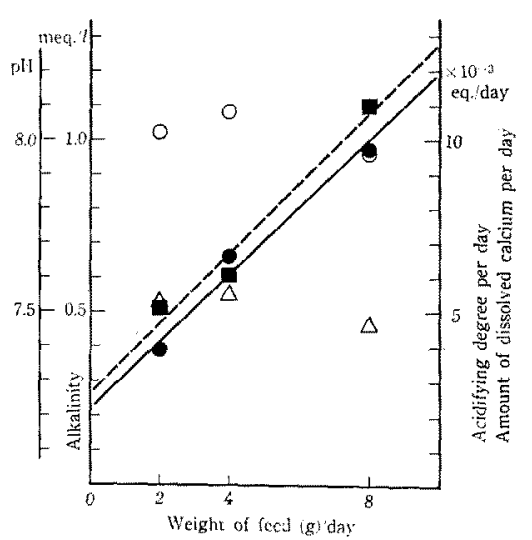

Fig. \% Relations of acidifying velocity and of the amount of dissolved calcium per day to feeding rate, and the levels of $\mathrm{pH}$ and alkalinity where they became constant. Open triangle and open circle represent respectively the levels of $\mathrm{pH}$ and alkalinity where they became constant. Closed circle represents the acidifying velocity of the aquarium water without coral (control test). Closed square represents the amount of dissolved calcium per day obtained from its daily increase after alkalinity became constant. 
with coral began to decrease due to dissolution of calcium. Thus, after 10 days, the $\mathrm{pH}$ value and the alkalinity value of this aquarium became almost constant at the levels of about 7.5 and about 1.02 meq. $/ l$, respectively.

In addition, the results of experiments in which the fishes were cultured with $2 \mathrm{~g}$ and $8 \mathrm{~g}$ of feed per day are shown in Fig. 7, together with the results of the above-mentioned experiment. Fig. 7 also shows the levels of $\mathrm{pH}$ and alkalinity where they became constant, the acidifying velocity of the aquarium water without coral (control) and the amount of dissolved calcium per day obtained from its daily increase after alkalinity became constant. In every experiment with the feeding rate of 2,4 , and $8 \mathrm{~g}$ per day, the $\mathrm{pH}$ value and the alkalinity value were all balanced at about 7.5 and at about 1.0 meq./ $l$, respectively. The relation of the acidifying velocity (eq./day) to the feeding rate (g/day) well coresponded with that of the rate of dissolution of calcium (eq./day) from coral to the feeding rate. This fact makes it clear that the prevention effect of coral on acidification of aquarium water is distinctly due to the dissolution of calcium from coral.

\section{Discussion}

It has been made clear that the acidifying velocity (eq./day) of aquarium water is so considerably high that the alkalinity value may be reduced from 2.0 (natural sea water) to nearly 0 meq. $/ l$, within only 20 days when black sea breams are cultured in $100 l$ of sea water by feeding with $10 \mathrm{~g}$ of shrimp per day. However, in the practical tank, it is usual that reduction of the $\mathrm{pH}$ value and the alkalinity value of the culture water is not so remarkable as calculated here, because of the dissolution of calcium from $\mathrm{CaCO}_{3}$ such as coral and shell contained in the filter sands. As to the concrete tank, it seems possible to prevent the culture water from acidification due to the dissolution of calcium from concrete. To ascertain this point, an experiment was conducted by using concrete block which had been soaked in a fresh water pond for two weeks. The result of this experiment suggested that the preventive effect of concrete on acidification might not at all be expected in spite of the abundant dissolution of calcium. The reason for this noteworthy point remains to be solved.

From the relation of the acidifying velocity to the feeding rate obtained in this study, it became possible to estimate the weight of lime required for neutrization of aquarium sea water prior to the actual addition.

\section{Summary}

Studies were made on acidification of the culture water in marine aquariums with closed circulating system in connection with the conditions of culturing fishes, and the results obtained were as follows. 
1) The acidifying velocity of aquarium water is not affected by the variation in grain size of the filter sands.

2) The relation between the acidifying velocity of aquarium water ( $V$ eq./day) and the feeding rate $(F \mathrm{~g} /$ day) can be represented by the following equation.

$$
V=0.92 F \times 10^{-3}
$$

3) The variation of body weight of fish cultured in an aquarium as compared with that of the feeding rate may not bring any significant difference in the acidifying velocity of the aquarium water.

4) The nitrifying ability of aquarium filter is exhibited well enough to keep ammonium- $\mathrm{N}$ at a low level even if the $\mathrm{pH}$ value becomes under 7.0.

5) Nitrification never ceases completely even if acidification ceases at the $\mathrm{pH}$ value under 4.8 and at the alkalinity value of nearly 0 meq. $/ l$.

6) The low values of $\mathrm{pH}$ and alkalinity hardly bring any harmful influence on cultured fish and so far as both values remain within a low level, ammonium- $\mathrm{N}$ accumulated in the sea water brings forth little influence.

7) A proper amount of $\mathrm{CaCO}_{3}$ such as coral and shell put in the aquarium, can maintain the value of $\mathrm{pH}$ and alkalinity over 7.5 and $1.0 \mathrm{meq} / l$, respectively, and its preventive effect on acidification of the culture water is distinctly due to the dissolution of calcium.

\section{Acknowledgement}

The author is pleased to acknowledge the continuing encouragement of Associate Prof. R. Hirano of Fac. of Agriculture, Univ. of Tokyo, and Prof. M. OKa of Fac. of Fisheries, Nagasaki Univ., and wishes to express his thanks to Mr. K. YAMANAGa for assistance in the performance of part of the experiments.

\section{References}

1) K. Hirayama: This bull., 31, 977-982, (1965).

2) K. Hirayama: This bull, 31, 983-990 (1965).

3) K. Hirayama: This bull., 32, 11-19 (1966).

4) K. Hirayama: This bull., 32, 20-27 (1966).

5) A. SAeKI: Bull. Mar. Biol. St. of Asamushi, Tôhoku Univ., 11, 99-103 (1963).

6) A. KaWAi, Y. YoshidA and M. Kimata: This bull., 31, 65-71 (1965). 